\title{
Design of a Discrete-time Output-feedback Based Repetitive-control System
}

\author{
Lan Zhou ${ }^{1} \quad$ Jin-Hua She ${ }^{2} \quad$ Min $\mathrm{Wu}^{3}$ \\ ${ }^{1}$ School of Information and Electrical Engineering, Hunan University of Science and Technology, Xiangtan 411201, China \\ ${ }^{2}$ School of Computer Science, Tokyo University of Technology, Tokyo 192-0982, Japan \\ ${ }^{3}$ School of Information Science and Engineering, Central South University, Changsha 410083, China
}

\begin{abstract}
This paper deals with the problem of designing a robust discrete output-feedback based repetitive-control system for a class of linear plants with periodic uncertainties. The periodicity of the repetitive-control system is exploited to establish a two-dimensional (2D) model that converts the design problem into a robust stabilization problem for a discrete 2D system. By employing Lyapunov stability theory and the singular-value decomposition of the output matrix, a linear-matrix-inequality (LMI) based stability condition is derived. The condition can be used directly to design the gains of the repetitive controller. Two tuning parameters in the LMI enable the preferential adjustment of control and learning. A numerical example illustrates the design procedure and demonstrates the validity of the method.
\end{abstract}

Keywords: Repetitive control, discrete two-dimensional system, singular-value decomposition, output feedback, linear matrix inequality (LMI).

\section{Introduction}

By repeating the same operation, a person gradually becomes skilled, and finally can operate with great efficiency and precision. In fact, the repetition of operation is a process of learning and gradual progress. Based on the internal model principle ${ }^{[1]}$, Inoue et al. ${ }^{[2]}$ added a human-like learning capability to a control system and devised a new control strategy called repetitive control (RC). Fig. 1 shows the configuration of a discrete-time repetitive-control system (RCS).

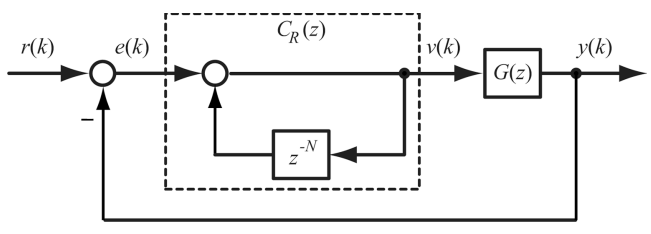

Fig. 1 Configuration of a basic discrete-time repetitive-control system

In Fig. $1, G(z)$ is a compensated plant, and $z$ is a shift operator. The part enclosed by the dotted line is a repetitive controller that contains a pure delay with a positivefeedback loop. For a given periodic reference input $r(k)$ with period of $N$, a repetitive controller gradually improves the tracking precision through repeated learning actions, which involve adding the control input of the previous period $v(k-N)$ to that of the present period to regulate the control input. As a result, the tracking error is reduced step

\footnotetext{
Manuscript received September 10, 2012; revised March 1, 2013

This work was supported by National Natural Science Foundation of China (Nos.61210011 and 61203010), National Science Fund for Distinguished Youth Scholars of China (No.60425310), Scientific Research Fund of Hunan Provincial Education Department (No.12B044), and Hunan Natural Science Foundation (No. 11JJ4059).
}

by step. Finally, the output tracks the reference input without any steady-state error. RC is similar to iterative learning control (ILC) ${ }^{[3]}$, which is another well-known method that makes use of the previous control trials. However, the difference between RC and ILC regarding the initial state of every period makes the issues of interest (stabilization, control system design, etc.) for these two methods very different.

An RCS has its inherent two-dimensional (2D) structural characteristics. It actually involves two different actions: Control within each repetition period and learning between periods. However, most analysis and design methods for an RCS developed for one-dimensional (1D) space ignore the difference between these two actions and only consider their overall effect. Consequently, they are incapable of making fundamental improvements in transient performance ${ }^{[4-6]}$. For the case of continuous RC, Zhou et al. ${ }^{[7,8]}$ employed $2 \mathrm{D}$ system theory ${ }^{[9,10]}$ to design a robust RCS. Mapping the RCS to $2 \mathrm{D}$ space yields a continuous-discrete $2 \mathrm{D}$ model. Unlike the 1D methods, the method in $[7,8]$ enables preferential adjustment of control and learning. The resulting control system exhibits both satisfactory robustness and good tracking performance.

RCS has been studied mainly in the continuous time domain. However, in practical control systems, digital implementation of repetitive controller is simpler than ana$\log$ counterpart. So, developing a design method for a discrete-time robust RCS has practical significance. References $[11,12]$ proposed a design method for discrete-time robust RCS, which is based on a discrete $2 \mathrm{D}$ model. It converted the system design to the robust stabilization problem for a discrete $2 \mathrm{D}$ system. However, the whole state of the plant is needed for the design of the controller, which is unfortunately unavailable in many practical applications. A design method that employs only the output of a plant is more practical. 
This paper presents a method of designing a discretetime robust RCS based on static output-feedback for a class of linear plants with periodic uncertainties. First, applying the lifting technique, a new discrete $2 \mathrm{D}$ model is established that accurately describes the characteristics of RCS and allows us to adjust control and learning preferentially. Then, by employing a 2D Lyapunov functional and the singularvalue decomposition (SVD) of the output matrix, a linearmatrix-inequality (LMI) based sufficient stability condition is derived. Two tuning parameters in the LMI are used to adjust control and learning. Finally, a numerical example demonstrates the effectiveness of the design method. Throughout this paper, $\mathbf{Z}^{+}$is the set of non-negative integers, $\mathbf{C}^{p}$ is the $p$-dimensional vector space over complex numbers, $\aleph$ is the linear space of all the functions from $\{0,1,2,3, \cdots, N-1\}$ to $\mathbf{C}^{p}, l_{2}\left(\mathbf{Z}^{+}, \mathbf{C}^{p}\right)$ is the linear space of square integrable functions from $\mathbf{Z}^{+}$to $\mathbf{C}^{p}$, and $l_{2}\left(\mathbf{Z}^{+}, \aleph\right)$ is the linear space of all the functions from $\mathbf{Z}^{+}$to $\aleph$.

\section{Problem description}

This paper considers the discrete-time RCS in Fig. 2. It is assumed that the process dynamics have been sampled by the zeroth-order hold method at a uniform rate $T_{s}$. The dynamics model of the repetitive controller $C_{R}(z)$ is

$$
v(k)= \begin{cases}e(k), & 0 \leqslant k<N \\ e(k)+v(k-N), & k \geqslant N\end{cases}
$$

where $e(k)=r(k)-y(k)$ is the tracking error.

In Fig. 2, the single-input single-output (SISO) compensated plant with a relative degree of zero and time-varying structured uncertainties is

$$
\left\{\begin{array}{l}
x(k+1)=(A+\delta A(k)) x(k)+(B+\delta B(k)) u(k) \\
y(k)=C x(k)+D u(k)
\end{array}\right.
$$

where $x(k) \in \mathbf{R}^{n}$ is the state of the plant, and $u(k) \in \mathbf{R}$ and $y(k) \in \mathbf{R}$ are the control input and output, respectively. A, $B, C$ and $D$ are real constant matrices. Assume that the structured uncertainties of the plant are

$$
\left[\begin{array}{ll}
\delta A(k) & \delta B(k)
\end{array}\right]=M F(k)\left[\begin{array}{ll}
N_{0} & N_{1}
\end{array}\right]
$$

where $M, N_{0}$ and $N_{1}$ are known constant matrices, and $F(k)$ is a real, unknown, and time-varying matrix with Lebesgue measurable elements satisfying

$$
F^{\mathrm{T}}(k) F(k) \leqslant I, \quad \forall k \in \mathbf{Z}^{+} .
$$

We make the following assumption.

Assumption 1. The uncertainties $\delta A(k)$ and $\delta B(k)$ vary periodically with the same period as that of the reference input $r(k)$, i.e.,

$$
\left\{\begin{array}{l}
\delta A(k+N)=\delta A(k) \\
\delta B(k+N)=\delta B(k)
\end{array}, \forall k \in \mathbf{Z}^{+} .\right.
$$

From (3) and (5), we have

$$
F(k+N)=F(k)
$$

The linear control law based on output feedback is

$$
u(k)=K_{e} v(k)+K_{y} y(k), \quad K_{e}, K_{y} \in \mathbf{R}
$$

where $K_{e}$ is the feedback gain of the repetitive controller and $K_{y}$ is the output feedback gain.

This paper considers the following design problem: Design suitable control gains $K_{e}$ and $K_{y}$ in (7) such that the RCS in Fig. 2 is robustly stable for the admissible uncertainties (3).

Equations (1), (2) and (7) describe the dynamics of the RCS in Fig. 2 in the time domain. Note that (7) can be rewritten as

$$
\begin{aligned}
u(k)= & K_{e}[e(k)+v(k-N)]+K_{y} y(k)= \\
& K_{e} v(k-N)+\left\{K_{e} r(k)+\left(K_{y}-K_{e}\right) y(k)\right\} .
\end{aligned}
$$

Equation (8) shows that the control law $u(k)$ contains two kinds of information: Information on the previous period (first term on the right-hand side) and information on the present period (second term).

Since we can view the past state as a kind of experience, $\mathrm{RC}$ actually involves two different types of actions: Control and learning. In other words, we use the information on the present and previous periods respectively to produce the present control input. A design method that enables independent adjustment of control and learning can potentially provide better transient performance. But from (8), we cannot preferentially adjust control and learning actions by directly changing the control gains $K_{e}$ and $K_{y}$ in (7). To solve this problem, we present a $2 \mathrm{D}$ description for the RCS in Fig. 2 in the following.

First, focusing on the periodicity of RC, we employ the lifting technique ${ }^{[13]}$ (Fig. 3) to slice the time axis into intervals of length $N$. That converts the vector-valued discretetime signal sequence $\{\xi(k)\}$ into the function-valued signal sequence $\left\{\xi_{i}(j)\right\}$. Its element is denoted as $\xi(i, j)$ in this paper. That is,

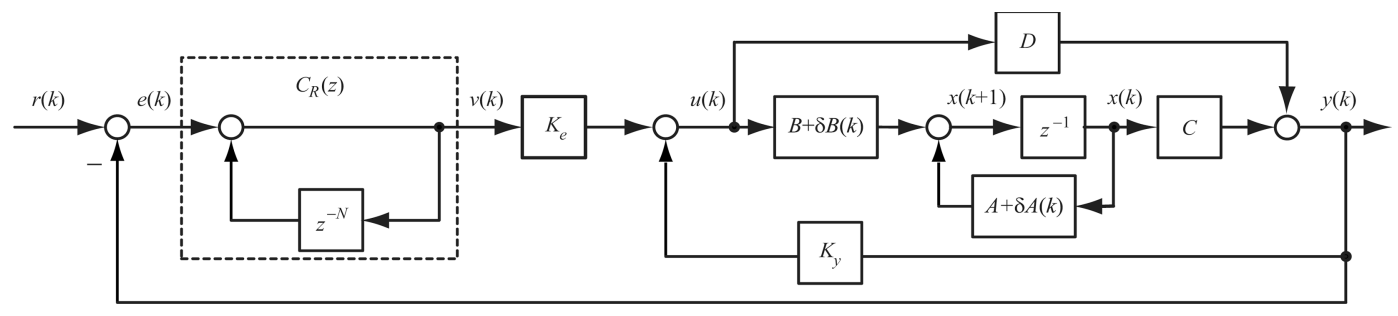

Fig. 2 Configuration of the discrete output-feedback-based repetitive-control system 

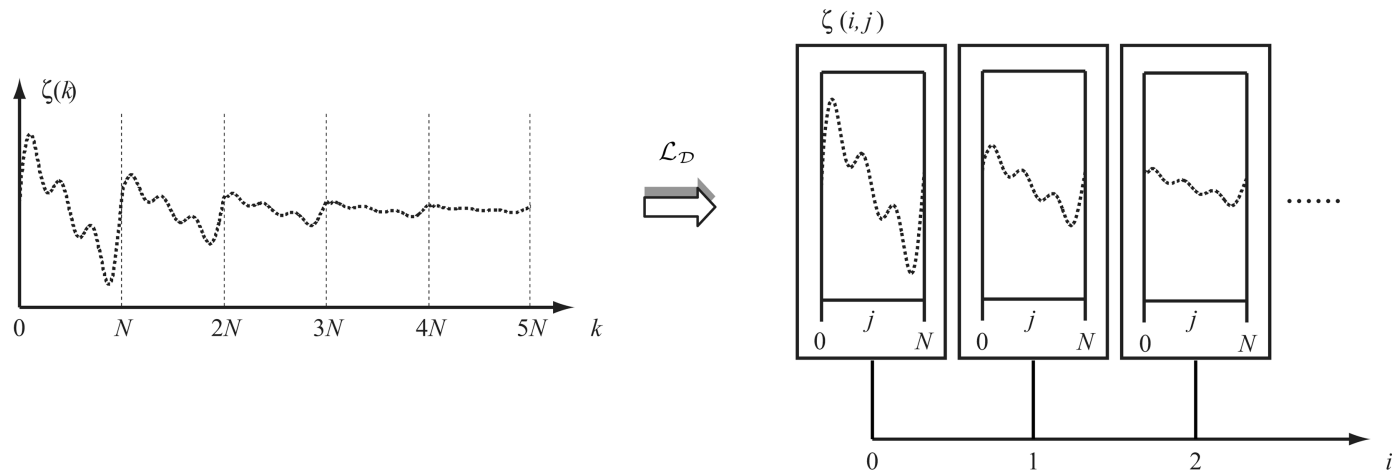

Fig. 3 Mapping $\mathcal{L}_{\mathcal{D}}$

$$
\begin{aligned}
\mathcal{L}_{\mathcal{D}}: & l_{2}\left(\mathbf{Z}^{+}, \mathbf{C}^{p}\right) \rightarrow l_{2}\left(\mathbf{Z}^{+}, \aleph\right) \\
& \xi(i, j)=\xi_{i}(j)=\mathcal{L}_{\mathcal{D}}(\xi(k)) \\
& k=i N+j, i \in \mathbf{Z}^{+}, j \in\{0,1,2,3, \cdots, N-1\}
\end{aligned}
$$

$\mathcal{L}_{\mathcal{D}}$ is an isometric, isomorphic transformation between $l_{2}\left(\mathbf{Z}^{+}, \mathbf{C}^{p}\right)$ and $l_{2}\left(\mathbf{Z}^{+}, \aleph\right)$.

Since the stability of the system does not depend on an exogenous signal, we set $r(k)=0$. Then, from (1), (2) and (8), we get the following $2 \mathrm{D}$ representation for the RCS in Fig. 2.

$$
\left\{\begin{array}{c}
x(i, j+1)=(A+\delta A(i, j)) x(i, j)+(B+\delta B(i, j)) u(i, j) \\
v(i, j)=-C x(i, j)-D u(i, j)+v(i-1, j)
\end{array}\right.
$$

with the 2D feedback control law of the form

$$
u(i, j)=F_{e} v(i-1, j)+F_{p} C x(i, j)
$$

where

$$
\begin{aligned}
F_{e} & =\frac{K_{e}}{1+\left(K_{e}-K_{y}\right) D} \\
F_{p} & =\frac{K_{y}-K_{e}}{1+\left(K_{e}-K_{y}\right) D} .
\end{aligned}
$$

Its dynamic boundary conditions are

$$
x(i+1,0)=x(i, N), \quad i \in \mathbf{Z}^{+} .
$$

In (9), the first equation describes the control action within one repetition period, and the second equation describes the learning behavior between two adjacent periods. We can adjust them preferentially by changing $F_{p}$ and $F_{e}$ in (10) and thereby accelerate the convergence of the tracking error. This is a big advantage over the 1D methods. Note that there is a coupling relationship between control and learning, and we cannot adjust them completely and independently. This can be observed from the second equation of (9). Meanwhile, from (11), the control gains in Fig. 2 can be rewritten as

$$
\begin{aligned}
K_{e} & =\frac{F_{e}}{1+D F_{p}} \\
K_{y} & =F_{e}+\frac{F_{p}\left(1-D F_{e}\right)}{1+D F_{p}} .
\end{aligned}
$$

Based on the 2D model (9), the design of the robust RCS in Fig. 2 is reformulated as the following problem: Find an admissible control law (10) for discrete 2D system (9) under boundary conditions of the form (12) such that the system is robustly stable and achieves fast convergence.

\section{Design of discrete output-feedback based RCS}

Substituting the control input (10) into (9) yields a representation of the $2 \mathrm{D}$ closed-loop RCS

$$
\delta \eta(i, j)=A_{l} \eta(i, j)+B_{l} \Gamma(i, j)
$$

where

$$
\begin{aligned}
\delta \eta(i, j) & =\left[\begin{array}{cc}
x^{\mathrm{T}}(i, j+1) & v^{\mathrm{T}}(i, j)
\end{array}\right]^{\mathrm{T}} \\
\eta(i, j) & =\left[\begin{array}{cc}
x^{\mathrm{T}}(i, j) & v^{\mathrm{T}}(i-1, j)
\end{array}\right]^{\mathrm{T}} \\
A_{l} & =\left[\begin{array}{cc}
A+B F_{p} C & B F_{e} \\
-C-D F_{p} C & 1-D F_{e}
\end{array}\right] \\
B_{l} & =\left[\begin{array}{c}
M \\
0
\end{array}\right] \\
\Psi & =\left[\begin{array}{cc}
N_{0}+N_{1} F_{p} C & N_{1} F_{e}
\end{array}\right] \\
\Gamma(i, j) & =F(i, j) \Psi \eta(i, j) .
\end{aligned}
$$

From (4),

$$
\Gamma^{\mathrm{T}}(i, j) \Gamma(i, j) \leqslant \eta^{\mathrm{T}}(i, j) \Psi^{\mathrm{T}} \Psi \eta(i, j) .
$$

Below, we derive a sufficient robust stability condition for the closed-loop 2D system (14) by constructing a $2 \mathrm{D}$ Lyapunov functional and combining the SVD of the output matrix.

Definition $\mathbf{1}^{[14]}$. Assume that the output matrix $C$ in (2) has full row rank and $\operatorname{rank}(C)=m$. The SVD of $C$ is

$$
C=U\left[\begin{array}{ll}
S & 0
\end{array}\right] V^{\mathrm{T}}
$$

where $S \in \mathbf{R}^{m \times m}$ is a diagonal matrix with positive, diagonal elements in decreasing order, $0 \in \mathbf{R}^{m \times(n-m)}$ is a zero matrix, $U \in \mathbf{R}^{m \times m}$ and $V \in \mathbf{R}^{n \times n}$ are unitary matrices.

For the SVD (17), the following lemma presents an equivalent condition for matrix equation $C X=\bar{X} C$.

Lemma $\mathbf{1}^{[15]}$. For a given $C \in \mathbf{R}^{m \times n}$ with $\operatorname{rank}(C)=$ $m$, if $X \in \mathbf{R}^{n \times n}$ is a symmetric matrix, then there exists a matrix $\bar{X} \in \mathbf{R}^{m \times m}$ such that $C X=\bar{X} C$ holds if and only if

$$
X=V \operatorname{diag}\left\{X_{11}, X_{22}\right\} V^{\mathrm{T}}
$$


where $X_{11} \in \mathbf{R}^{m \times m}$ and $X_{22} \in \mathbf{R}^{(n-m) \times(n-m)}$.

The following two lemmas are also employed in the derivation of the stability condition for the 2D system (14).

Lemma 2 (Schur complement ${ }^{[16]}$ ). For any real matrix $\Sigma=\Sigma^{\mathrm{T}}$, the following assertions are equivalent:

1) $\Sigma=\left[\begin{array}{cc}S_{11} & S_{12} \\ * & S_{22}\end{array}\right]<0$

2) $S_{11}<0$ and $S_{22}-S_{12}^{\mathrm{T}} S_{11}^{-1} S_{12}<0$

3) $S_{22}<0$ and $S_{11}-S_{12} S_{22}^{-1} S_{12}^{\mathrm{T}}<0$

Lemma 3 (S-procedure ${ }^{[17]}$ ). Let $\Omega_{0}(x)$ and $\Omega_{1}(x)$ be two quadratic matrix functions over $\mathbf{R}^{n}$, and let $\Omega_{1}(x) \leqslant 0$ for all $x \in \mathbf{R}^{n}-\{0\}$. Then, $\Omega_{0}(x)<0$ holds for all $x \in \mathbf{R}^{n}-\{0\}$ if and only if there exists an $\varepsilon \geqslant 0$ such that

$$
\Omega_{0}(x)-\varepsilon \Omega_{1}(x)<0, \quad \forall x \in \mathbf{R}^{n}-\{0\} .
$$

The above lemmas lead to Theorem 1 .

Theorem 1. For given positive scalars $\alpha$ and $\beta$, if there exist symmetric, positive-definite matrices $X_{11}, X_{22}, X_{2}$, and arbitrary matrices $W_{1}$ and $W_{2}$ such that the LMI

$$
\left[\begin{array}{cccccc}
-\alpha X_{1} & 0 & 0 & \Theta_{14} & \Theta_{15} & \Theta_{16} \\
* & -\beta X_{2} & 0 & \Theta_{24} & \Theta_{25} & \Theta_{26} \\
* & * & -I & M^{\mathrm{T}} & 0 & 0 \\
* & * & * & -\alpha X_{1} & 0 & 0 \\
* & * & * & * & -\beta X_{2} & 0 \\
* & * & * & * & * & -I
\end{array}\right]<0
$$

holds, where

$$
\begin{aligned}
& X_{1}=V \operatorname{diag}\left\{X_{11}, X_{22}\right\} V^{\mathrm{T}} \\
& \Theta_{14}=\alpha X_{1} A^{\mathrm{T}}+\alpha C^{\mathrm{T}} W_{1}^{\mathrm{T}} B^{\mathrm{T}} \\
& \Theta_{15}=-\alpha X_{1} C^{\mathrm{T}}-\alpha C^{\mathrm{T}} W_{1}^{\mathrm{T}} D^{\mathrm{T}} \\
& \Theta_{16}=\alpha X_{1} N_{0}^{\mathrm{T}}+\alpha C^{\mathrm{T}} W_{1}^{\mathrm{T}} N_{1}^{\mathrm{T}} \\
& \Theta_{24}=\beta W_{2}^{\mathrm{T}} B^{\mathrm{T}} \\
& \Theta_{25}=\beta X_{2}-\beta W_{2}^{\mathrm{T}} D^{\mathrm{T}} \\
& \Theta_{26}=\beta W_{2}^{\mathrm{T}} N_{1}^{\mathrm{T}}
\end{aligned}
$$

then the discrete RCS (14) in Fig. 2 is asymptotically stable. And the 2D feedback gains in (10) are

$$
\begin{aligned}
& F_{e}=W_{2} X_{2}^{-1} \\
& F_{p}=W_{1} U S X_{11}^{-1} S^{-1} U^{\mathrm{T}}
\end{aligned}
$$

where $U$ and $S$ are defined in SVD (17).

Proof. Let

$$
P_{1}=X_{1}^{-1}, \quad P_{2}=X_{2}^{-1} .
$$

Choose a Lyapunov functional candidate to be

$$
V(i, j)=V_{1}(i, j)+V_{2}(i, j)
$$

where

$$
\begin{aligned}
& V_{1}(i, j)=x^{\mathrm{T}}(i, j) \frac{1}{\alpha} P_{1} x(i, j) \\
& V_{2}(i, j)=v^{\mathrm{T}}(i-1, j) \frac{1}{\beta} P_{2} v(i-1, j) .
\end{aligned}
$$

Consider the associated increment $\Delta V(i, j)$ as

$\Delta V(i, j)=V_{1}(i, j+1)-V_{1}(i, j)+V_{2}(i+1, j)-V_{2}(i, j)$.
Along the time trajectory of (14), we have

$$
\Delta V(i, j)=\tilde{\eta}^{\mathrm{T}}(i, j)\left(-P+\hat{A}_{l}^{\mathrm{T}} P \hat{A}_{l}\right) \tilde{\eta}(i, j)
$$

where

$$
\begin{aligned}
& \tilde{\eta}^{\mathrm{T}}(i, j)=\left[\begin{array}{lll}
x^{\mathrm{T}}(i, j) & v^{\mathrm{T}}(i-1, j) & \Gamma^{\mathrm{T}}(i, j)
\end{array}\right] \\
& P=\operatorname{diag}\left\{\frac{1}{\alpha} P_{1}, \quad \frac{1}{\beta} P_{2}, \quad 0\right\} \\
& \hat{A}_{l}^{\mathrm{T}}=\left[\begin{array}{ccc}
A^{\mathrm{T}}+C^{\mathrm{T}} F_{p}^{\mathrm{T}} B^{\mathrm{T}} & -C^{\mathrm{T}}-C^{\mathrm{T}} F_{p}^{\mathrm{T}} D^{\mathrm{T}} & 0 \\
F_{e}^{\mathrm{T}} B^{\mathrm{T}} & 1-F_{e}^{\mathrm{T}} D^{\mathrm{T}} & 0 \\
M^{\mathrm{T}} & 0 & 0
\end{array}\right]
\end{aligned}
$$

Furthermore, (15) and (25) yield

$$
\begin{gathered}
\Delta V(i, j)-\left[\Gamma^{\mathrm{T}}(i, j) \Gamma(i, j)-\eta^{\mathrm{T}}(i, j) \Psi^{\mathrm{T}} \Psi \eta(i, j)\right]= \\
\tilde{\eta}^{\mathrm{T}}(i, j) \Lambda \tilde{\eta}(i, j)
\end{gathered}
$$

where

$$
\begin{aligned}
& \Lambda=-\tilde{P}+\tilde{A}_{l}^{\mathrm{T}} \tilde{P} \tilde{A}_{l} \\
& \tilde{P}=\operatorname{diag}\left\{\frac{1}{\alpha} P_{1}, \quad \frac{1}{\beta} P_{2}, \quad I\right\} \\
& \tilde{A}_{l}^{\mathrm{T}}=\left[\begin{array}{ccc}
\tilde{A}_{l 11}^{\mathrm{T}} & \tilde{A}_{l 12}^{\mathrm{T}} & \tilde{A}_{l 13}^{\mathrm{T}} \\
F_{e}^{\mathrm{T}} B^{\mathrm{T}} & 1-F_{e}^{\mathrm{T}} D^{\mathrm{T}} & F_{e}^{\mathrm{T}} N_{1}^{\mathrm{T}} \\
M^{\mathrm{T}} & 0 & 0
\end{array}\right] \\
& \tilde{A}_{l 11}^{\mathrm{T}}=A^{\mathrm{T}}+C^{\mathrm{T}} F_{p}^{\mathrm{T}} B^{\mathrm{T}} \\
& \tilde{A}_{l 12}^{\mathrm{T}}=-C^{\mathrm{T}}-C^{\mathrm{T}} F_{p}^{\mathrm{T}} D^{\mathrm{T}} \\
& \tilde{A}_{l 13}^{\mathrm{T}}=N_{0}^{\mathrm{T}}+C^{\mathrm{T}} F_{p}^{\mathrm{T}} N_{1}^{\mathrm{T}} .
\end{aligned}
$$

Combining (16) and (26), and applying S-procedure (Lemma 3) to (26), we find that if $\Lambda<0$, then for any $\tilde{\eta}(i, j) \neq 0, \Delta V(i, j)<0 ;$ thus the closed-loop system $(14)$ is asymptotically stable.

Also, from Schur complement Lemma $2, \Lambda<0$ is equivalent to the following matrix inequality

$$
\Xi=\left[\begin{array}{cccccc}
-\frac{1}{\alpha} P_{1} & 0 & 0 & \Xi_{14} & \Xi_{15} & \Xi_{16} \\
* & -\frac{1}{\beta} P_{2} & 0 & \Xi_{24} & \Xi_{25} & \Xi_{26} \\
* & * & -I & \Xi_{34} & 0 & 0 \\
* & * & * & -\frac{1}{\alpha} P_{1} & 0 & 0 \\
* & * & * & * & -\frac{1}{\beta} P_{2} & 0 \\
* & * & * & * & * & -I
\end{array}\right]<0
$$

where

$$
\begin{aligned}
& \Xi_{14}=\frac{1}{\alpha}\left(A^{\mathrm{T}}+C^{\mathrm{T}} F_{p}^{\mathrm{T}} B^{\mathrm{T}}\right) P_{1} \\
& \Xi_{15}=\frac{1}{\beta}\left(-C^{\mathrm{T}}-C^{\mathrm{T}} F_{p}^{\mathrm{T}} D^{\mathrm{T}}\right) P_{2} \\
& \Xi_{16}=N_{0}^{\mathrm{T}}+C^{\mathrm{T}} F_{p}^{\mathrm{T}} N_{1}^{\mathrm{T}} \\
& \Xi_{24}=\frac{1}{\alpha} F_{e}^{\mathrm{T}} B^{\mathrm{T}} \\
& \Xi_{25}=\frac{1}{\beta}\left(1-F_{e}^{\mathrm{T}} D^{\mathrm{T}}\right) P_{2} \\
& \Xi_{26}=F_{e}^{\mathrm{T}} N_{1}^{\mathrm{T}} \\
& \Xi_{34}=\frac{1}{\alpha} M^{\mathrm{T}} P_{1} .
\end{aligned}
$$


Since $X_{1}=V \operatorname{diag}\left\{X_{11}, X_{22}\right\} V^{\mathrm{T}}$, from Lemma 1, there exists $\bar{X}_{1}$ such that

$$
C X_{1}=\bar{X}_{1} C
$$

and

$$
\bar{X}_{1}^{-1}=U S X_{11}^{-1} S^{-1} U^{\mathrm{T}}
$$

Define

$$
W_{1}=F_{p} \bar{X}_{1}, \quad W_{2}=F_{e} X_{2} .
$$

Pre-multiplying and post-multiplying the matrix on the left-hand side of (27) by

$$
\operatorname{diag}\left\{\alpha X_{1}, \beta X_{2}, I, \alpha X_{1}, \beta X_{2}, I\right\}
$$

and substituting (28) and (30) into the corresponding matrix, we have LMI (18).

So, if LMI (18) holds, then the discrete closed-loop RCS (14) is robustly stable for the admissible uncertainties (3).

Finally, we obtain (20) from (29) and (30).

Remark 1. Theorem 1 provides an LMI-based sufficient robust stability condition for discrete $2 \mathrm{D}$ system (9) under control law (10). The condition can be easily used to directly design the controller parameters of the RCS in Fig. 2 using the feasp solver in robust control toolbox ${ }^{[18]}$. The two tuning parameters $\alpha$ and $\beta$ in LMI (18) manipulate the preferential adjustment of control and learning. More specifically, $\alpha$ adjusts the weighting matrix $P_{1}$ in $V_{1}(i, j)$, and $\beta$ adjusts the weighting matrix $P_{2}$ in $V_{2}(i, j)$. Note that $V_{1}(i, j)$ and $V_{2}(i, j)$ are quadratic terms related directly to control and learning. Accordingly, $\alpha$ and $\beta$ regulate the feasible solutions $F_{p}$ and $F_{e}$ in (20), respectively.

In addition, from Theorem 1, we can derive a sufficient stability condition for the nominal discrete $2 \mathrm{D}$ closed-loop system

$$
\delta \eta(i, j)=A_{l} \eta(i, j)
$$

where $\delta \eta(i, j), \eta(i, j)$ and $A_{l}$ are defined in (14).

Corollary 1. For given positive scalars $\alpha$ and $\beta$, if there exist symmetric, positive-definite matrices $X_{11}, X_{22}, X_{2}$, and arbitrary matrices $W_{1}$ and $W_{2}$ such that the following LMI holds

$$
\left[\begin{array}{lccc}
-\alpha X_{1} & 0 & \tilde{\Theta}_{13} & \tilde{\Theta}_{14} \\
* & -\beta X_{2} & \beta W_{2}^{\mathrm{T}} B^{\mathrm{T}} & \tilde{\Theta}_{24} \\
* & * & -\alpha X_{1} & 0 \\
* & * & * & -\beta X_{2}
\end{array}\right]<0
$$

where $X_{1}$ is defined in (19), and

$$
\begin{aligned}
& \tilde{\Theta}_{13}=\alpha X_{1} A^{\mathrm{T}}+\alpha C^{\mathrm{T}} W_{1}^{\mathrm{T}} B^{\mathrm{T}} \\
& \tilde{\Theta}_{14}=-\alpha X_{1} C^{\mathrm{T}}-\alpha C^{\mathrm{T}} W_{1}^{\mathrm{T}} D^{\mathrm{T}} \\
& \tilde{\Theta}_{24}=\beta X_{2}-\beta W_{2}^{\mathrm{T}} D^{\mathrm{T}}
\end{aligned}
$$

then the $2 \mathrm{D}$ system (31) is asymptotically stable. And the $2 \mathrm{D}$ feedback gains in (10) are

$$
\begin{aligned}
& F_{e}=W_{2} X_{2}^{-1} \\
& F_{p}=W_{1} U S X_{11}^{-1} S^{-1} U^{\mathrm{T}} .
\end{aligned}
$$

\section{Numerical example}

Consider the problem of designing a controller for a direct current motor driven manipulator with a proportional integral regulator. The control input is the voltage applied to the armature, and the output is the rotational torque of the manipulator. The state-space model of the motor can be described using (2). Assume that the parameters of the uncertain plant (2) are

$$
\left\{\begin{array}{l}
A=\left[\begin{array}{cc}
-0.93 & 0 \\
0.3 & -0.85
\end{array}\right], \quad B=\left[\begin{array}{c}
0 \\
0.6
\end{array}\right] \\
C=\left[\begin{array}{ll}
1 & 0
\end{array}\right], \quad D=2 \\
M=\left[\begin{array}{ll}
1 & 0 \\
0 & 1
\end{array}\right], \quad N_{0}=\left[\begin{array}{ll}
0 & 0.01 \\
0 & 0.01
\end{array}\right] \\
N_{1}=\left[\begin{array}{c}
0 \\
0.05
\end{array}\right], \quad F(k)=\left[\begin{array}{cc}
\sin \pi k & 0 \\
0 & \sin \pi k
\end{array}\right]
\end{array}\right.
$$

and the sampling frequency is $f_{s}=\frac{1}{T_{s}}=10 \mathrm{~Hz}$.

We consider the problem of tracking the reference input

$$
r(k)=\sin \pi k+0.5 \sin 2 \pi k+0.5 \sin 3 \pi k
$$

and then the repetition period of the RCS in Fig. 2 is

$$
N=\frac{2}{T_{s}}=20
$$

Choose the performance index

$$
J_{5}=\frac{1}{2 f_{s}} \sum_{i=0}^{4} \sum_{j=1}^{20} e^{2}(i N+j)
$$

as a criterion for the selection of the tuning parameters $\alpha$ and $\beta$. Fig. 4 shows the effect of adjusting the tuning parameters through simulation on three parameter sets:

$$
\left\{\begin{array}{lll}
\text { (a): } & \alpha=0.6, \quad \beta=0.05 \\
\text { (b): } & \alpha=0.6, \quad \beta=0.13 \\
\text { (c): } & \alpha=0.2, \quad \beta=0.13 .
\end{array}\right.
$$

The performance indices are

$$
J_{5}^{a}=3.3208, \quad J_{5}^{b}=0.8068, \quad J_{5}^{c}=0.1841 .
$$

Note that, in Fig. 4, the tracking error in the first period shows only the control performance because there is no learning behavior within this period, and the convergence speeds of the tracking error in different periods show different learning efficiencies. Comparing the simulation results of sets (a) and (b), it is clear that the tracking speed is accelerated by tuning $\beta$ from 0.05 to 0.13 . And comparing the simulation results of sets (b) and (c), we know that the control performance is greatly improved by tuning $\alpha$ from 0.6 to 0.2 . These simulation results show that adjusting $\alpha$ can mainly affect control, and adjusting $\beta$ can mainly affect learning. Note that, due to the coupling relationship between control and learning, tuning $\alpha$ and $\beta$ also strongly influences learning and control, respectively. This can be observed from Fig. 4. Among the three parameter sets, (c) 
provides the best overall control and learning performance. In this way, we use the optimization algorithm

$$
\min J_{5} \quad \text { s.t. LMI (18) }
$$

to calculate the best tuning parameters.

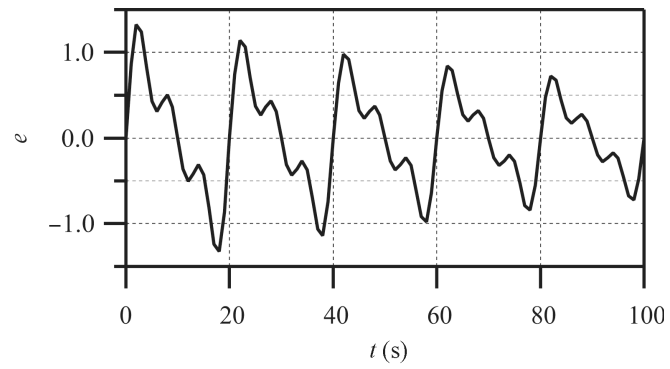

(a) $\alpha=0.6, \beta=0.05$

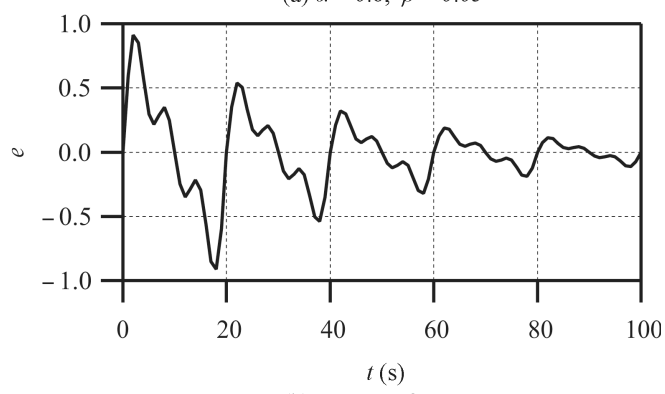

(b) $\alpha=0.6, \beta=0.13$

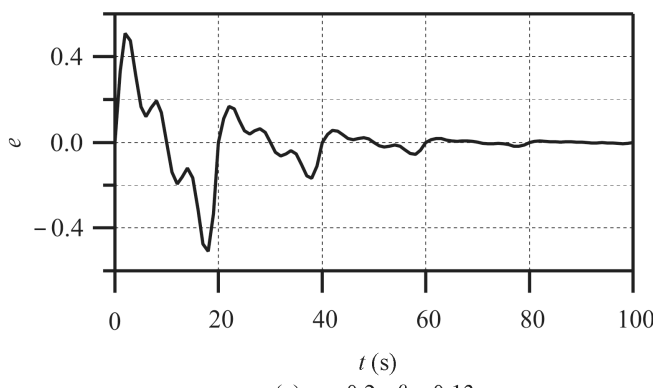

(c) $\alpha=0.2, \beta=0.13$

Fig. 4 Tracking errors for parameter sets (38)

A combination of (40) and the fixed-step method in the ranges

$$
\alpha \in(0,1], \quad \beta \in(0,1]
$$

yields the best parameters set

$$
\alpha=0.2, \beta=0.99 \text {. }
$$

From Theorem 1, we obtain the corresponding control gains

$$
K_{e}=68.9590, \quad K_{y}=-2.9357 .
$$

Simulation results in Fig. 5 show that the system is asymptotically stable for the admissible uncertainties and it enters into the steady state in the third period. Moreover, $J_{5}=0.0034$.

Based on the above analysis, we present a design algorithm for the output-feedback-based RCS in Fig. 2.

\section{Algorithm 1.}

Step 1. Use (40) to find $\alpha$ and $\beta$ that minimize $J_{5}$.

Step 2. Use Theorem 1 to calculate $F_{e}$ and $F_{p}$.

Step 3. Use (13) to calculate $K_{e}$ and $K_{y}$.
In addition, using the guaranteed cost algorithm in [11], we carried out simulation (in Fig. 6) for the uncertain plant (34). We found that $J_{5}^{[11]}=0.4248$. The comparison of the simulation results (Figs. 5 and 6 ) shows that the preferential adjustment of control and learning greatly improves the transient performance.

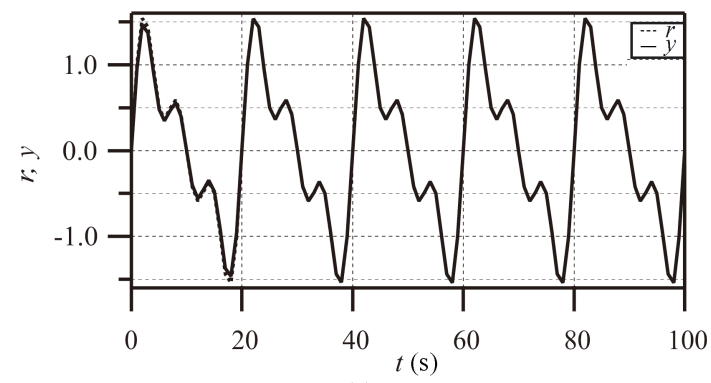

(a) $r$ and $y$

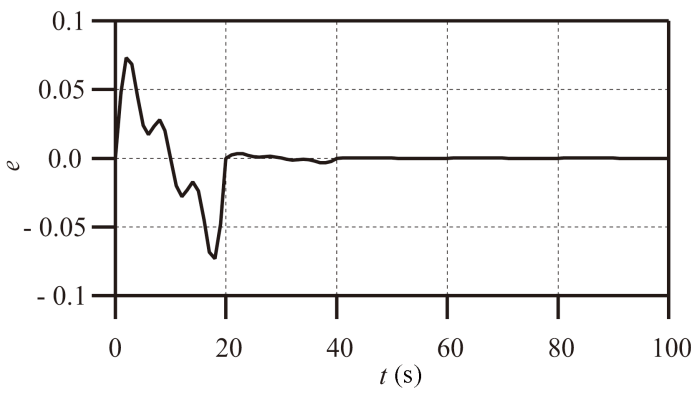

(b) Tracking error

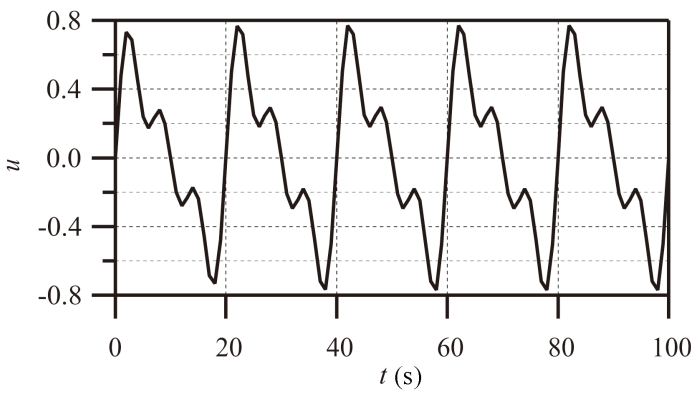

(c) Control input

Fig. 5 Simulation results for output-feedback-based RCS for $\alpha=0.2$ and $\beta=0.99$

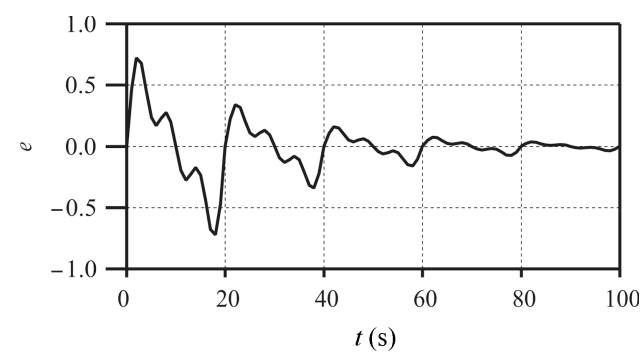

Fig. 6 Tracking error using the method in [11]

\section{Conclusions}

This paper described an LMI-based design method for a discrete-time robust RCS based on static output-feedback for a class of linear plants with periodic uncertainties. Exploiting the inherent $2 \mathrm{D}$ structural characteristics of $\mathrm{RC}$, we 
established a discrete 2D model, making it possible to preferentially adjust control and learning by means of the gains in the 2D control law. The stability theory of $2 \mathrm{D}$ systems and the SVD of the output matrix were applied to derive an LMI-based sufficient stability condition for the closed-loop system. The two tuning parameters in the condition manipulated the preferential adjustment of control and learning. Finally, a numerical example illustrated the design and tuning procedures, and simulation results demonstrated the effectiveness of the method.

\section{References}

[1] B. A. Francis, W. M. Wonham. The internal model principle for linear multivariable regulators. Applied Mathematics and Optimization, vol. 2, no. 2, pp. 170-194, 1975.

[2] T. Inoue, M. nakano, S. Iwai. High accuracy control of a proton synchrotron magnet power supply. In Proceedings of the 8th Internat Fedaration on Automatic Control, Kyoto, Japan, pp. 216-221,1981.

[3] D. Li, J. M. Li. Adaptive iterative learning control for nonlinearly parameterized systems with unknown time-varying delay and unknown control direction. International Journal of Automation and Computing, vol.9, no. 6, pp. 578-586, 2012.

[4] M. Tomizuka, T. C. Tsao, K. K. Chew. Analysis and synthesis of discrete-time repetitive controllers. Journal of Dynamic Systems, Measurement, and Control, vol.111, no. 3 , pp. 353-358, 1989.

[5] J. Na, R. Costa-Castelló, R. Griñó, X. M. Ren. Discretetime repetitive controller for time-delay systems with disturbance observer. Asian Journal of Control, vol. 14, no. 5, pp. 1340-1354, 2012.

[6] Y. D. Han, D. H. Owens, B. Chu. Discrete time linear optimal repetitive control - A low order controller scheme. In Proceedings of the 2nd International Conference on Computational Intelligence, Communication Systems and Networks, IEEE, Washington, DC, USA, pp. 238-243, 2010.

[7] L. Zhou, J. She, M. Wu, J. Zhang. Design of robust modified repetitive-control system for linear periodic plants. Journal of Dynamic Systems, Measurement, and Control, vol. 134, no. 1, pp. 011023-1-7, 2012.

[8] M. Wu, L. Zhou, J. She. Design of observer-based $H_{\infty}$ robust repetitive-control system. IEEE Transactions on $\mathrm{Au}$ tomatic Control, vol. 56, no. 6, pp. 1452-1457, 2011.

[9] N. K. Bose. Multidimensional Systems Theory and Applications, The Netherlands: Kluwer Academic Publishers, 2003.

[10] J. Shi, F. R. Gao, T. J. Wu. Robust design of integrated feedback and iterative learning control of a batch process based on a 2D Roesser system. Journal of Process Control, vol. 15, no. 8, pp. 907-924, 2005.

[11] C. H. Fu, Y. He, M. Wu, S. G. Yuan. Guaranteed cost robust discrete repetitive control based on two-dimension model. In: Proceedings of the 29th Chinese Control Conference, IEEE, Beijing, China, pp. 2023-2028, 2010. (in Chinese)

[12] S. G. Yuan. M. Wu, B. G. Xu, R. J. Liu. Design of discretetime repetitive control system based on two-dimensional model. International Journal of Automation and Computing, vol. 9, no. 2, pp. 165-170, 2012.

[13] Y. Yamamoto. A function space approach to sampled data control systems and tracking problems. IEEE Transactions on Automatic Control, vol. 39, no. 4, pp. 703-713, 1994.

[14] K. M. Zhou, J. C. Doyle, K. Glover. Robust and Optimal Control, Prentice Hall, pp. 32-35, 1996.

[15] D. W. C. Ho, G. P. Lu. Robust stabilization for a class of discrete-time non-linear systems via output feedback: The unified LMI approach. International Journal of Control, vol. 76, no. 2, pp. 105-115, 2003.
[16] P. P. Khargonek, I. R. Petersen, K. M. Zhou. Robust stabilization of uncertain linear systems: Quadratic stabilizability and $H_{\infty}$ control theory. IEEE Transactions on $\mathrm{Au}$ tomatic Control, vol. 35, no. 3, pp. 356-361, 1990.

[17] V. A. Yakubovich. S-procedure in Nonlinear Control Theory, Vestnik Leningrad University, vol. 1, pp. 62-77, 1971.

[18] G. Balas, R. Chiang, A. Packard, M. Safonov. Robust Control Toolbox-User's Guide, 3. MA: The MathWorks, Inc, 2005.

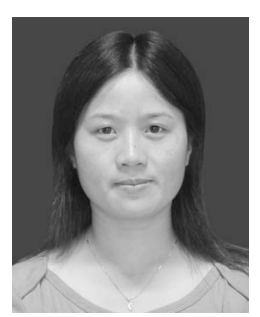

Lan Zhou received the B. Sc. degree from Hunan Normal University, China in 1998 and the M.Sc. degree from Central South University, China in 2006. From 2008 to 2010, she was a joint cultivation doctoral candidate of Japan and China. She received her $\mathrm{Ph}$. D. degree in control science and engineering from Central South University, China in 2011. She is an associate professor of control theory and control engineering with the School of Information and Electrical Engineering, Hunan University of Science and Technology, China.

Her research interests include robust control, repetitive control, and control application.

E-mail: zlly98@yahoo.cn (Corresponding author)

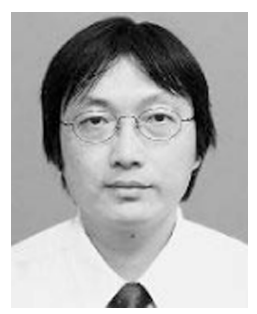

Jin-Hua She received the B.Sc. degree in engineering from Central South University, China in 1983 , the M. Sc. and the Ph. D. degrees in engineering from the Tokyo Institute of Technology, Japan in 1990 and 1993. In 1993, he joined the Department of Mechatronics, School of Engineering, Tokyo University of Technology, Japan. In 2008, he transferred to the School of Computer Science, Tokyo University of Technology, where he is currently a professor. He received the Control Engineering Practice Paper Prize of the International Federation of Automatic Control (IFAC) in 1999 (jointly with M. Wu and M. Nakano).

His research interests include the application of control theory, repetitive control, process control, internet-based engineering education, and robotics.

E-mail: she@stf.teu.jp.cn

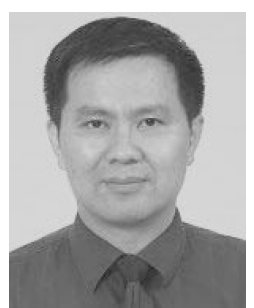

Min Wu received the B. Sc. and M. Sc. degrees in engineering from Central South University, China in 1983 and 1986, respectively, and the $\mathrm{Ph}$. D. degree in engineering from Tokyo Institute of Technology, Japan in 1999. Since 1986, he has been a faculty member with Central South University, where he is currently a professor of automatic control engineering with the School of Information Science and Engineering. He was a visiting scholar at the Department of Electrical Engineering, Tohoku University, Japan from 1989 to 1990, a visiting research scholar at the Department of Control and Systems Engineering, Tokyo Institute of Technology, Japan from 1996 to 1999, and a visiting professor at the School of Mechanical, Materials, Manufacturing Engineering and Management, University of Nottingham, UK from 2001 to 2002. He received the Control Engineering Practice Paper Prize of the International Federation of Automatic Control (IFAC) in 1999 (jointly with M. Nakano and J. She). He is a member of the Nonferrous Metals Society of China and the China Association of Automation.

His research interests include robust control and its application, process control, and intelligent control.

E-mail: min@csu.edu.cn 